\title{
XLIV. The attachment of quartz fibres
}

\section{V. Boys A.R.S.M. F.R.S.}

To cite this article: C. V. Boys A.R.S.M. F.R.S. (1894) XLIV. The attachment of quartz fibres , Philosophical Magazine Series 5, 37:228, 463-467, DOI: 10.1080/14786449408620573

To link to this article: http://dx.doi.org/10.1080/14786449408620573

$$
\text { 曲 Published online: } 08 \text { May } 2009 .
$$

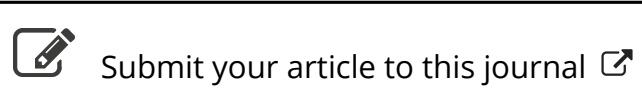

\footnotetext{
Џll Article views: 3
}

Q View related articles $\square$

Citing articles: 4 View citing articles $\sqsubset$ 


\section{$\left[\begin{array}{ll}463 & ]\end{array}\right.$ \\ XLIV. The Attachment of Quartz Fibres. By C. V. BoYs, A.R.S.M., F.R.S.*}

\section{M}

EMBERS of the Physical Society may remember that in $1887+$ I described a method of making fine fibres of glass and other materials, but especially of melted quartz, which latter had properties of great value, rendering them more suitable for experimental work of combined delicacy and accuracy than those of other known materials. Experiments made since by others as well as myself have further shown that for delicate work of the highest degree of accuracy they are essential.

The method of fastening them, however, at their ends to the pointed end of the torsion-pin at the top or of the suspension below by shellac varnish, or better by melted shellac, is apt to give rise, more especially if the fibre is unskilfully laid in place so that it is twisted round the point, to a slow creeping of the point of rest due to slow changes in the shellac. This, except for the first few days, can hardly ever be of an amount to seriously affect any observations; in fact I bave made many observations of the effect of gravitation between small masses with fibres so fastened of a great degree of accuracy, besides those with the radiomicrometer, pocket electrometer, \&c., without any inconvenience, yet I have felt that some method of attaching them which would be less likely to hold the fibre by a part in a state of torsional strain or of flexure would be preferable. If the part of the fibre held could certainly be in its natural position and state with respect to the rest, then, even if the fastening should fail to be as perfect as a true weld, any resulting change of zero should be small compared to that observed where the portion held is twisted or much bent.

The process of silvering, electro-coppering, and soldering is an obvious one, but it is not so easily carried out with a fair degree of certainty and in a manner which is convenient of application, as might be expected. My experience of last autumn has enabled ine to perform the process in a series of operations, each simple enough, and, as far as I am able to test it in the apparatus with which $I$ an now measuring the Newtonian constant of gravitation (which I may say is of unusual delicacy), with perfect success. In this case the fibre is necessarily stretched to not far from its breaking weight,

* Communicated by the Physical Society : read February 23, 1894.

+ Phil. Mag. June 1887. 
and it is in such cases that the stability of the fastening is most severely tried.

The first thing I found was that it was a mistake to solder the fibre to the torsion-rod and to the suspension directly. The difficulty of the manipulation is great and a change of fibre is very troublesome. The preferable plan is to solder the ends of the fibre to little tags of metal so small and light that they may be picked up by the fibre from anything on which they rest without risk of snapping the fibre at the point of junction. These tags, which are conveniently made of copper-foil, five millimetres long and one millimetre wide about at the wide end, tapering nearly to a point, can afterwards be fastened to the torsion support and the suspension by shellac varnish or by melted shellac, and now the enormous surface and the stiffness of the foil is sufficient to prevent any trouble from the causes to which reference has already been mado.

These tags might also for some purposes, either or both of them, be made of T-form to hang in a pair of V's, and so dispense with cement altogether, and allow of the easy interchange of suspensions or of fibres, but $I$ bave not myself employed such a form.

The following operations are those which $I$ have found to answer :-

1. Select a fibre of the right diameter to give the desired torsion. Since the torsion depends on the fourth power of the diameter, a small change in the diameter makes a fourfold change in the torsion, and great accuracy of measurement is needed where an exact torsional rigidity is required. Cut off a piece from two to three centimetres longer than will ultimately be required.

2. Fasten to the extreme ends of the fibre, with melted shellac, little weights of gold or platinum heavy enough to pierce a liquid surface.

3. Hang the fibre over a fixed round horizontal rod of wood, 1 centimetre in diameter or thereabouts, so that the little weights hang side by side, and lift up from below a little glass of strong nitric acid, so as to wet and clean the fibre well above the final points of attachment. The vessel must be wide enough to prevent capillarity from drawing the fibres to one side, or it must be brimful so that the surface is convex, which with nitric acid is objectionable. The vessel must be moved both upwards and downwards past the place at which the weights pass through the surface very rapidly, practically with a jerk; otherwise the weights will be drawn together by capillarity, and the fibres will get twisted. 
or capillarity will give trouble somehow. With the rapid movement the little weights hardly acquire any pendular motion.

4. After a minute or two do the same, but to a slightly greater depth, with water which may be distilled.

5 . When the acid may be supposed to be washed off, immerse in the same way in Rochelle salt silvering-solution (Kohlrausch, 'Physical Measurements,' p. 115).

6. Wash as in 4 .

7. Fill a glass with the copper solution that is employed in electrolytic measurements of current, i.e. not saturated, and slightly acid. Dip the extreme point of the positive wire from a single cell into the liquid, and with a clean smooth negative wire take the hanging ends, one at a time, and having made the contact outside the glass by resting the upper part of the silver coat upon the wire, let down into the solution, keeping the fibre in gentle movement on the wire and making it dip more and less in the liquid. In a few seconds the little weight will be bright red, and the immersed portion of the silvered coat will be bright red also. The silver coat has sufficient resistance to prevent unduly rapid deposition. Do the same to the other end.

8. Cut off to length, allowing about 5 millimetres at each end for the junction. Take tags of copper-foil three or four centimetres long and three or four millimetres wide, tapering to a point, and having tinned the pointed end of each with the minimum of solder, again wet with chloride-of-zinc solution. On the wet surface lay the coppered end, taking care that it points in the right direction. Capillarity will now hold it. Rapidly heat the copper to the melting-point by holding a point about one centimetre from the narrow end over a minute flame. The solder will flash and envelope the coppered fibre. Cut off the tag of the desired length, holding the metal by the tag with a pair of pliers and not by the heavy end.

9. Wash in boiling - water as in 4 to remove chloride of zinc. The fibre is now attached, but the protruding silver and copper give a want of definiteness in the place of attachment.

10. Dip up to the point of the tag in melted beeswax, following the precautions given in 3 , but the two tags may be more conveniently dipped separately.

11. Dip up to the top of the copper and silver in strong nitric acid.

12. Wash in boiling water, which removes acid and beeswax and leaves the fibre ready for use. 
13. If it is required to conduct electricity, as for instance to keep the needle of a quadrant electrometer electrically connected with a battery, the whole may now be silvered in a long tube and washed, otherwise it will insulate most perfectly. It may be mentioned here that for the most delicate possible electrometer, as I found in my experiments on the pocket electrometer, it is useless to expect to find any stability where a liquid surface is pioreed. The only method of communicating with the needle is through a silvered quartz fibre. Owing to the insulating quality of a clean quartz fibre, delicate experiments are apt to be disturbed by unintended electrification of the suspension, and this may still remain after means have been employed to prevent it, for mere metallic contact between different metals leaves the surfaces in effect at different potentials, depending on the metals used, and, as I showed, in an idiostatic instrument the disturbance due to platinum and zinc is many hundred times the least that can be detected.

I have sought to reduce this form of error by either or both of two methods. In the first I make the inside of the chamber surrounding the suspension a figure of revolution, the axis being the line of the fibre; in the other, when possible, I make the surfaces of the suspension and of the enclosure one and the same, preferably electro-gilt.

The first method in very small instruments also in the main avoids what can no longer be sifely neglected, as it has hitherto nearly always been, viz. gravitational attraction.

There is one more point which may be of some interest. If an unsilvered quartz fibre is threaded through a small hole in a thin metal plate, stretched by a suspended weight, and the hole is then wetted with chloride of zinc and soldered up, the fibre will, after washing off the fused chloride of zinc, pull out, leaving a hole fine and beautifully circular.

It is unnecessary to say more than $I$ have already done, on more than one occasion, on the necessity for making the free space round a suspension in any instrument of extreme delicacr as small as possible and enclosing it by massive metal, itself protected from outside heating and cooling by a non-conducting cover, such as $I$ have in the radio-micrometer; otherwise the convection currents set up in the free space will blow the suspension about, and produce vagaries which might be easily attributed to the fibre or its attachment. The disturbances due to this cause are apt to be much greater than anyone would at first imagine, and the small trouble spent in aroiding them in the manner indicated is well rewarded.

With regard to the manipulation with fine fibres. I have 
Method of finding the Refractire Index of a Liquid. 467 already pointed out that the darkness inside a drawer just pulled out, if the operator is sitting at a table in front of a window with a good light, is such that fine fibres can readily be seen upon it as a background. No velvet or smoked surface or artificial blackness of any lind is comparable with it. On such backgrounds fine fibres are to all intents and purposes invisible. What is in many respects preferable to the dark background, at least in certain operations, is a plain lookingglass lying on the table. Fibres resting upon it become intensely brilliunt and visible, provided the eye is so placed as not to see the sky light itself reflected from the mirror. One method of making the fibres very easily visible without influencing their torsion, is to smoke them with burning magnesium or arsenic. I do not suggest arsenic, but I mention it because of the very beautiful effect I once observed, after destroying all life in a small hot-house by burning a large quantity of bengal fire in which orpiment is a considerable constituent. All the spider-webs remained perfect with the spiders in their places as though alive, and the webs were of a dazzling white but perfect in form, undragged by the weight of the white arsenic upon them, thus contrasting strongly with the catenary distorted webs so much admired in frosty weather. It was this observation that suggested the magnesium smoking.

These last few points hardly come directly under the title of this paper, but I thought them worth adding as bearing upon the successful design of apparatus in which the full limit of delicacy and accuracy obtainable by the quartz fibre may be obtained, and upon the practical details of its treatment.

XLV. A Method of finding the Refractive Index of a Liquid; applicable when the Liquid is not Homogeneous. By T. H. LITTLEWOOD, M.A.*

Apparatus required.

THE chief piece of apparatus required for the method is a 1 telescope with fixed wires in the eyepiece, arranged so as to be capable of motion along a horizontal scale, without changing its inclination to the vertical. The horizontal motion can be measured either by a vernier or by a micrometer-screw.

* Communicated by the Physical Society : read February 23, 1804. 